Voix et Images

voixetimages

\title{
Le moi et ses doubles
}

Lucie Robert

Volume 35, numéro 1 (103), automne 2009

\section{Herménégilde Chiasson}

URI : https://id.erudit.org/iderudit/038578ar

DOI : https://doi.org/10.7202/038578ar

Aller au sommaire du numéro

\section{Éditeur(s)}

Université du Québec à Montréal

\section{ISSN}

0318-9201 (imprimé)

1705-933X (numérique)

Découvrir la revue

Citer ce compte rendu

Robert, L. (2009). Compte rendu de [Le moi et ses doubles]. Voix et Images, 35(1), 141-146. https://doi.org/10.7202/038578ar d'utilisation que vous pouvez consulter en ligne.

https://apropos.erudit.org/fr/usagers/politique-dutilisation/ 
D R A M A T U R G I E

Le moi et ses doubles

$++$

LUCIE ROBERT

Université du Québec à Montréal

Est-ce l'économie ou est-ce le besoin d'identification qui motive la crise du moi dans les textes dramatiques récents? L'économie, peut-être un peu, parce que cette crise donne naissance à des petites pièces, pingres de moyens et de propos, à des pièces brèves le plus souvent, n'exigeant qu'un décor minimal et un ou deux, parfois trois comédiens, au plus. L'identification aussi, sans doute, quand l'auteur dramatique replace son écriture au cœur de l'esthétique thêâtrale et qu'il se met à réfléchir à sa démarche, créant des personnages qui ne peuvent être que des copies inversées de lui-même ou des représentations fantomatiques de ses angoisses. Faux monologues, instantanés, projections concentrées sur un moment particulièrement révélateur, comme les quelques heures qui précèdent la mort, les pièces dont il est question ici ont en commun d'explorer divers procédés de dédoublement, révélant une scission entre le moi et l'autre, son double. Pour un auteur dramatique, cet autre est parfois l'acteur; pour l'acteur, il est souvent le spectateur. Tous ces autres contribuent à la construction du moi qui forcément leur résiste. Aussi arrive-t-il que le personnage perde son identité voire son unicité : il se multiplie ou se dissout; il se répète, refait un geste, réitère une parole, revit, mais dans un autre lieu voire dans un autre temps. Tout se passe comme si le moi était peut-être celui qui avait survécu à la présence de l'autre.

Rien ne saurait mieux rendre compte de ce type de théâtre que la pièce de Larry Tremblay, Le problème avec moi ${ }^{1}$, produite par le thêâtre Omnibus et créée en novembre 2007 à l'Espace Libre dans une mise en scène de Francine Alepin, puis reprise en avril et mai 2009 au Théâtre Périscope à Québec. Non seulement Larry Tremblay donne-t-il une seconde vie au personnage de Léo, que nous connaissions déjà par Le déclic du destin (1989), mais il lui adjoint, comme en miroir, un sosie quasi

$$
++
$$

1 Larry Tremblay, Le problème avec moi. Thêâtre, Carnières, Lansman, coll. «Nocturnes Théâtre», 2007, 34 p. 
homonyme, Léø. Dans la préface, Tremblay explique: «Dans le premier texte, je pose cette question: "qu'arrive-t-il quand il y a trop de moi pour un corps?" Dans le second, celle-ci: "qu'arrive-t-il quand il y a trop de corps pour un moi ?" Ces deux questions, au fond, ne font qu'une. Cependant, le théâtre permet de s'en amuser.» (5) Léo et Léø entrent donc en scène vêtus de la même manière, portant des accessoires semblables, mais ils ne se connaissent pas. Ils viennent tout juste de se rencontrer, chacun croyant que l'autre lui a volé son sac (puisque les sacs sont évidemment identiques). Peu à peu, ils se découvrent non sans une certaine inquiétude, se mirant l'un dans l'autre, mais (et comme dans une glace) à l'envers l'un de l'autre. Car Léo et Léø ne sont pas la même personne, comme si le clonage avait eu lieu la veille ou le matin et que depuis, leurs destins s'étaient légèrement séparés: le premier est plus ancré dans la réalité quotidienne, plus heureux aussi, le second, un peu mythomane et du genre inquiet; le premier a écrit aux journaux, le second a oublié. Ainsi de suite. Progressivement, la rencontre trouve ses modèles et ses images récurrentes, tel le personnage interprété par Antony Perkins dans le Psycho de Hitchcock, qui est lui-même un peu le docteur Jekyll et Mr Hyde. Léo et Léø se révèlent ainsi des doubles inversés. Le bonheur du premier soulève l'ire du second qui témoigne d'une certaine violence: «Tu as le don de m'énerver.» (23) Dans une finale digne de La cantatrice chauve, les deux personnages, toujours en miroir, «sortent du sac une robe qu'ils endossent. Puis ils sortent du sac une perruque de vieille femme, des lunettes, des pantoufles. Quand ils ont terminé leur transformation, ils sortent du sac un couteau de boucherie. Ils lèvent leur bras meurtrier. La musique cesse.» (34) Que se passera-t-il ? L'un deux va-t-il éliminer l'autre ou vont-ils simplement répéter leur rencontre, cette fois sous une autre apparence, peut-être même une nouvelle identité?

Le moi qui s'affiche dans Me, myself et moi-même. L'autobiographie, que vous n'aviez pas demandée ${ }^{2}$ de Stéphane E. Roy est celui de l'écrivain en difficulté, hésitant sur ce qu'il doit écrire, mais qui trouve là, en même temps, l'occasion de réinventer sa vie. Le préfacier, Gilbert Turp, insiste sur le caractère rédempteur de ce passage à vide qui «amène aussi la métaphore de la possibilité de réécrire sa vie, de laisser une trace, de signaler qu'on participe à l'Histoire [...] et qui permet que la détresse se renverse en espoir» (9). Toutefois, comme le titre le laisse entendre, $M e$, myself et moi-même repose sur la rencontre non pas entre deux, mais entre trois personnages: Stéphane l'écrivain, Emmanuel, son alter ego dix ans plus jeune, et Gilbert, l'acteur qui doit interpréter le rôle une fois la pièce écrite. Ce dernier n'est pas dupe : «faut le faire $[. .$.$] écrire une pièce où les deux personnages principaux sont toi-même»$ (19). La pièce n'est pas exempte de certains poncifs du genre quand le personnage le plus jeune, Emmanuel, se montre écœuré de ce que Stéphane est devenu, assagi

$$
++
$$

2 Stéphane E. Roy, Me, myself et moi-même. L'autobiographie, que vous n'aviez pas demandée. Une pièce égocentrique, préface de Gilbert Turp, Montréal, Dramaturges éditeurs, 2007, 115 p. 
voire embourgeoisé, un père de famille responsable qui a renoncé à l'alcool et aux joies des nuits de bamboche: «La maturité, c'est la mort de l'artiste.» (66) Ou encore, autre poncif, quand l'acteur qu'est Gilbert s'englue dans une certaine forme de cabotinage, invente des tirades et résiste au texte que lui écrit Stéphane avant de se résigner: "C'est toi qui écris, c'est toi qui choisis. Moi je subis. C'est le terrible sort des comédiens.» (31) Il reste que cette rencontre entre les deux âges de l'écrivain sonne l'heure des bilans. Il faut alors reconstituer l'Histoire, déterminer le moment où tout a basculé: "Les attentats, ça secoue quelqu'un» (81), explique d'abord Stéphane, qui dans un même souffle, apprend à Emmanuel la mort de leur mère et la maladie de leur père, avant de lui faire rencontrer le petit William, à qui la pièce est d'ailleurs dédiée. Pièce créée le 5 juillet 2006 au Théâtre Arts Station de MontSaint-Hilaire, dans une mise en scène de l'auteur, Me, myself et moi-même présente une version masculine de la difficile relation entre la création et la procréation: «mon gars me fait retrouver des souvenirs enfouis. Parce que... un souvenir qui n'est pas raconté, réactivé par la parole... meurt.» (77)

La paternité est aussi au cœur de ce curieux texte qu'est Terre océane ${ }^{3}$ de Daniel Danis, texte qui ne se présente pas dans le dispositif graphique habituel des textes dramatiques et qui est entrecoupé de plusieurs photographies de Susan Coolen. Celles-ci montrent des éléments appartenant à l'histoire naturelle, agencés de manière muséographique: plumes, brindilles, feuilles, à l'occasion, un jouet. Sous sa forme dramatique, la pièce a été créee en octobre 2007 au Thêâtre d'Aujourd'hui, dans une mise en scène de Gil Champagne, et reprise au Trident à Québec en janvier 2008. Elle paraît ici sous la forme d'un récit, un récit dialogué, mais un récit tout de même en ce qu'il repose presque entièrement sur un point de vue unique, celui d'Antoine, qui témoigne comme au nom des autres. Le texte est d'ailleurs sous-titré «roman-dit», comme l'était la récente e (2006).

On sonne. Le lendemain de son quarantième anniversaire, Antoine ouvre la porte sur son fils, Gabriel. C'est apparemment à son tour de s'occuper de cet enfant qu'il avait autrefois adopté avec Mireille et qu'il n'a jamais revu depuis leur séparation. Il lui faut s'organiser d'urgence, surtout que Gabriel est malade, un cancer dont il est en rémission, du moins le croit-il, car le médecin est formel: six à neuf mois au mieux. Père et fils s'installent alors à la campagne, chez l'oncle Dave, où les trois générations vont être réunies par cet état d'attente qui précède l'événement inéluctable qu'est la mort de Gabriel. Il ne se passe rien d'autre dans ce récit tout entier structuré par les quelques moments heureux que vivent les personnages qui se découvrent l'un à l'autre, l'un à travers l'autre. Antoine peut ainsi tracer le bilan de son enfance à lui, mais peut-être aussi la revit-il un peu, auprès de cet oncle vaguement sorcier chez qui son père l'avait placé autrefois, un jour qu'il «était si enragé

$$
+++
$$

3 Daniel Danis, Terre océane. Récit, photographies de Susan Coolen, Montréal, Dazibao, coll. «Des photographes", 2008, 112 p. 
qu'il avait eu peur de [le] tuer» (29). Le plus jeune, Gabriel, va donc mourir quelque temps après Noël, le plus âgé, Dave, comprend qu'il le suivra de peu. Restera alors seul l'homme du milieu, Antoine, qui entre-temps aura appris de son fils à remettre ses valeurs à l'endroit: «Jusqu'aux portes des autres mondes, il m'aura obligé à être attentif à l'autre, à des réels qui me renvoient à mes propres nuances.» (109) Il y a dans ce nouveau texte de Daniel Danis un rappel de ses précédents, Cendres de cailloux, Le chant du dire-dire, en ce que les personnages sont encore marqués par une sorte de violence initiatrice. Cependant, on y trouve surtout la grande douceur ferme qui caractérise l'individu réinscrit dans son histoire, voire dans l'épopée de son temps, et qui, de là, entend se réapproprier sa vie, ce qu'on lisait aussi dans e (2006), premier texte marquant ce changement dans le registre d'écriture de l'auteur.

Emprunté à l'anglais «floating ice», le mot floes, qui donne son titre à la pièce de Sébastien Harrisson ${ }^{4}$, désigne «les petites îles, nées des fractures de la banquise qui flottent au gré du courant» (7), emportant parfois un ours polaire égaré ou un explorateur. Créée au Thêâtre d'Aujourd'hui, le 28 février 2001, dans une mise en scène d'Alice Ronfard, Floes, pièce écrite en vers et sans ponctuation, met en scène deux personnages, Adrien, professeur de littérature retraité, et Paul, médecin retraité, tous deux âgés de 70 ans. Cette nuit-là, quelque part au large de l'Alaska, un petit biplan s'est abîmé, laissant ses deux passagers sur la banquise. Paul qui, d'expérience, connaît tout du Nord, «Des infimes détails de l'hypothermie/Jusqu'à la glaciation des âmes» (20), est résigné à mourir là et il écoute les voix; Adrien n'accepte pas et attend les secours. L'heure est aux bilans. Soudain on entend une voix de femme et une silhouette se dessine dans une lumière pâle. Adrien espère. Paul doute et rappelle: "Il y a une légende/Selon laquelle/Les voix en Arctique ne se perdent pas/Selon laquelle/Elles restent prisonnières de l'architecture de la banquise/Leur écho se heurtant d'iceberg en iceberg/Parfois durant des centaines d'années/Avant de trouver une oreille qui les entende.» (30) Arrive en effet Lady Franklin, jeune veuve de l'explorateur John Franklin, vêtue d'un costume de voyage du XIX siècle, et enceinte depuis cent ans, qui cherche son mari. Métaphore de cette mort inéluctable, Lady Franklin saisit d'abord Paul qu'elle emporte avec elle. Curieuse pièce que cette Floes, conçue autour de bruits (le chant de l'ourse, la plainte de Lady Franklin) plutôt qu'avec des images, et dont l'action se déroule dans la nuit polaire, qui oppose la chaleur humanitaire du médecin (qui meurt sans regrets) à la dureté froide du professeur de littérature, "Ce stupide métier» (48), lequel, resté seul, comprend soudainement ce qu'est l'éternité.

Floes est suivie D'Alaska, pièce créée le 20 mars 2007 à L'Arrière Scène, Centre dramatique pour l'enfance et la jeunesse à Belœil, dans une mise en scène de Frédéric Dubois. Celle-ci met en scène deux personnages, Madame, 70 ans, et Aujourd'hui, 14 ans. S'ils sont réunis dans ce pavillon de banlieue, c'est que le

$$
++
$$

4 Sébastien Harrisson, Floes et D’Alaska. Suite nordique, Montréal, Dramaturges éditeurs, 2007, 151 p. 
meilleur (le seul) ami d'Aujourd'hui, Dominique, est parti vivre au Nevada avec sa famille et que la conjointe de Madame est partie faire une croisière en Alaska avec une de ses étudiantes. À la question «Dis-moi plutôt ce que tu viens faire dans mon salon...», l'adolescent répond: «Te faire parler.» (85) Aujourd'hui est en fugue et Madame sait qu'elle va mourir. Chacun doit parvenir à raconter son histoire. À la fin, le jardin se couvre de neige, puis d'une flaque d'eau: le jardin est devenu mer du Nord avec des icebergs et des oiseaux. Aujourd'hui est devenu un homme et Madame est morte.

À première vue, William Dubé ne paraît pas être en crise. À 50 ans, il est fonctionnaire de l'État français, contrôleur de train, en poste sur le Thalys Bruges-Paris. $C^{\prime}$ est justement dans ce train, assis sur une banquette pendant sa pause, que William Dubé rencontre des passagers avec lesquels il lie conversation. Ce jour-là, le livre de lecture du passager ramène le contrôleur "près de trente ans en arrière, à [s]on premier voyage en Europe» (13). Après le vol entre Montréal et Amsterdam, il avait pris le train pour Paris, et avait alors rencontré un homme : "pardon/you may stay" (19). Coincé entre deux langues, le voyageur d'alors avait remarqué: "c'est le Canada, ça, le Canada des deux langues qui se boudent, qui se font la gueule, et faire un pas dans la langue de l'autre, ça non, jamais» (17). La conversation s'était donc déroulée d'abord en allemand puis, parce que le voyageur n'était pas allemand non plus, elle s'était poursuivie en français, mais à sens unique. Car le destinataire de ces mots répond peu: «Il me dit qu'il est auteur.» (19) Deux ans plus tard paraissait un roman, intitulé La rumeur du monde est sans beauté, écrit par l'homme du train. C'est ce livre que lit le passager trente ans plus tard. Celui-ci se rend en Roumanie par affaires. Émerge alors un deuxième souvenir: vingt ans plus tôt, «j'ai donné, moi aussi, en matière de trafic avec la Roumanie/une histoire de faux passeports » (23). Il y aura un troisième souvenir, celui d'une femme qui s'est jetée du cinquième étage de son hôtel.

Le caractère inquiétant du personnage apparaît plus nettement quand il montre à l'inconnu ses trois passeports: «c'est à croire que j'aspire qu'à ça, me faire prendre, avec mes passeports que j'étale, comme ça, sans précaution» (55). Depuis trente ans, en effet, William Dubé vit avec de faux papiers obtenus en Roumanie, qui le nomment Richard Dubé, le font citoyen français et lui permettent de travailler à bord des trains européens: "depuis toujours je me sens en sursis» (49). Puis, un inconnu lui avait offert le passeport, un passeport allemand, au nom de Wilhelm Stouffer, nom du mari de la femme de l'hôtel. Aujourd'hui pourtant, il hésite et il raconte. Quelle est l'importance de ces trois identités, de ces trois passeports qui se valent bien l'un l'autre puisque William n'a pas celui qu'il voudrait? Je suis d'un would be pays ${ }^{5}$ est d'ailleurs le titre de ce monologue de François Godin, lui aussi écrit en vers et sans ponctuation, créé au Théâtre d'Aujourd'hui le 4 septembre 2007

$$
+++
$$

5 François Godin, Je suis d'un would be pays. Théâtre, Montréal, Leméac, coll. «Thêâtre», 2008, 67 p. 
sous la direction de Gervais Gaudreault. Et si l'action se passe dans un train, c'est bien sûr que les trains européens sont la copie inversée des «trains canadiens [qui ne] sont là que pour nous faire croire au Canada» (24).

« Dans une maison immense, il y a, quelque part, un escalier dérobé. En haut de cet escalier, il y a un couloir étroit. Au bout du couloir étroit, il y a une porte close. Devant la porte close, il y a une jeune femme, Grâce, qui regarde, comme hypnotisée.» (5) Telle est la didascalie initiale de la plus récente pièce de Carole Fréchette, La petite pièce en haut de l'escalier ${ }^{6}$, résultat d'une commande du Theâtre CAF National de Bretagne-Rennes, créée au Théâtre du Nouveau Monde le 4 mars 2008, dans une mise en scène de Lorraine Pintal. Dès cette première image, l'on songe à Barbe-Bleue. En effet, par son mariage - qui est aussi le troisième d'Henri -, Grâce mène la vie de princesse dont sa mère avait toujours rêvé, à l'opposé de sa sœur Anne, qui en est l'antithèse. Que va-t-elle faire maintenant de son temps? «Je vais m'occuper, je vais créer. - Anne : Créer quoi ? - Grâce : Je ne sais pas. De la beauté en tout cas. Tout le monde rêve de ça, avoir du temps pour créer.» (10) Or, Henri, le mari de Grâce, lui a interdit d'entrer dans la petite pièce: «J'aimerais mieux que tu y ailles pas, c'est tout. [...] Parce que... c'est plein de cadavres.» (15) Peu à peu se font jour un certain nombre de faits inquiétants. Grâce ne peut résister à l'envie de voir la petite pièce et paraît attirée par elle de manière obsessive. Selon sa sœur, elle pleure tous les soirs avant de dormir. Personne ne connaît le passé d'Henri et celuici harcèle Grâce au téléphone. Même Jenny, la bonne, adopte un comportement étrange, épiant Grâce, acceptant ses cadeaux, mais cherchant les faux pas dans l'espoir peut-être que sa loyauté envers Henri lui vaudra quelque récompense. Arrive ce qui devait arriver. Grâce entre dans la pièce, où elle aperçoit un sac d'où sort un gémissement: «Un homme qui saigne.» (30) À ce moment précis, l'action de la pièce se dérègle. Henri survient et la surprend. Jenny lui offre une hache, de l'alcool, ce qu'il veut. En bas, Jocelyne, la mère de Grâce qui vient d'arriver, attend et s'inquiète. Nous aussi. Puis, l'action recommence, mais autrement: Grâce est dans son bain quand arrive Henri.

«Il y a pas de conte» (57), affirme Henri qui, à propos de l'homme de la petite pièce, explique enfin: «Je sais pas qui il est, d'où il vient, de l'autre bout du monde, des entrailles de cette maison. Je sais seulement qu'il est là, et il a besoin de mes larmes, de tes larmes peut-être, de vraies larmes.» (72-73) Comme dans chaque pièce précédente de Carole Fréchette, une phrase itérative donne sens au texte: «qu'est-ce que c'est des vraies larmes? ? Accessoirement, reste la question qui nous hante: qui est cet homme? Qui est-il en effet sinon la représentation des peurs que Grâce a enfin eu le courage d'affronter, peut-être aussi celle des terreurs d'Henri, voire la grâce qui permettra à ces deux êtres de se rencontrer enfin?

$$
+++
$$

6 Carole Fréchette, La petite pièce en haut de l'escalier, Arles/Montréal, Actes Sud-Papiers/Leméac, 2008, $74 \mathrm{p}$. 